\title{
The Research of Design of Human Resource Recruitment System Based on the Total Relationship Flow Management Theorems
}

\author{
Fangling Hu \\ School of Management, Jinan University, Guangzhou, China \\ Email: guyue2758@163.com
}

Received 18 September 2014; revised 20 October 2014; accepted 17 November 2014

Academic Editor: Denis Morin, Université du Québec à Montréal, Canada

Copyright (C) 2014 by author and Scientific Research Publishing Inc.

This work is licensed under the Creative Commons Attribution International License (CC BY). http://creativecommons.org/licenses/by/4.0/

\section{c) (i) Open Access}

\begin{abstract}
With the development of knowledge economy, organizational strategic resource is more than the physical production such as capital, and it also includes the human resources characterized by skills, knowledge and intelligence. Recruitment as the first part of introducing talents, its quality directly influences the effect of introducing talents, more related to the long-term development of the enterprise. Therefore, building perfect recruitment system is not only an important part of the enterprise to get the resource, but also the key to support enterprise strategy implementation. Based on the total relationship flow management theory, this paper put forward suggestions to build an perfect recruitment system though designing the behaviors of the recruitment system, determining the appropriate relationship flow, and paying attention to time delay and system maintenance.
\end{abstract}

Keywords

Total Relationship Flow Management Theory, System Designing, Recruitment System

\section{Introduction}

With the increased level of awareness of the importance of the talent, some companies are fully aware of the use of the recruitment and try their best to rely on the more professional, scientific management methods to optimize the recruitment process, and to improve the recruitment effect. Managers find that optimizing the recruitment system can not only increase the efficiency, better shape corporate image and then be able to attract more talent. 
However, we also find it difficult to ignore the problems in many enterprises, especially small and medium enterprises that exist in recruitment. For example, many enterprises follow the principle of "the best" rather than "optimal" in the selection process, so they are blind pursuit of highly educated and elite background. Some HR departments are lack of internal communication and coordination. These problems not only lead to recruiting costs rising and dissatisfied recruitment result, but also lead to decrease in the efficiency of the entire enterprise. In view of this, establishing a perfect recruitment system is not just the HR department's priority, but also an issue that concerns the entire organization.

\section{Literature Review}

Human resources as the most important strategic resource has attract scholars and business managers' attention. Some academic point the HR recruitment emerge, but the angles of these research are not the same. By studying literature I find two types of research about recruitment: section studies and systems research. Section research focuses on how to make the selection of talent, how to test the candidates on the list and which test methods we should use. As in 1960 the book "Career Decision" which is published by the American career guidance experts Hollander (John L. Holland) put forward the personality theory proposed career match. And domestic scholars Yongjiang Han (2007) proposed to use of analytic hierarchy process (AHP) to improve recruitment decisions [1].

In 1940s, the famous biologist V. L. Bertalanffy founded the general systems theory which aims to study the common principles and rules that the variety of different systems obey in the different subject field [2]. Over half a century, the general systems theory has been a noticeable field. Accordingly, the human resource management system becomes more and more outstanding scholars' object. Based on systems theory, The Human Resource Management Human Resource Management is defined by Wright (1991) as the input, processing and output of the system. Xiaobing Zhang (2009) summarized the definition of human resource management system on the basis of previous research. He thought human resource management system have several common ground: 1) The activities, functions and processes that organizations use to attract, develop and maintain human resource; 2) These human resource management practices have consistent or complementary; 3) Organization through the implementation of the management practices achieve organizational goals. Recruitment system is an important part of human resource management system [3].

According to these researches, we can regard the recruitment system means as that the organization attract, identify and recruit talents through certain plans, channels and programs. However, existing studies on the recruitment system are based on case studies or experience and don't have strong theoretical support. This paper attempts to make up for the deficiencies in the existing research under the guidance of the total relationship flow management theorems.

\section{The Total Relationship Flow Management Theorems}

Professor F. Lin proposed and developed a general systems theory: The structural theory of general systems, which are faced with problems in mathematical expression develops the general systems theory into precise theoretical content [4]. This theory is thought with the height to effectively to solve practical system problem.

The total relationship management theory is put forward by Professor Lin based of the structural theory of general systems. The theorem states that the behavior of complex systems only depend on the input relationship flow and relationship flow set $R f_{\text {ord }}(t)$ (the total relationship flow), especially the relationship cycle in the system [5]. These relationships include information flow, energy flow, matter flow, fund flow, personal flow, etc. Therefore, in order to better understand and control the behavior of complex systems, we should understand and control the flow of their total relationship.

\subsection{Concepts and Theorem}

Relationship flow $R_{i j}(t)$ involves the action factor part $e(i)$ one $(j)$ at the time tin the system. Part $e(i)$ have an effect on the part of $e(j)$ through the effect factor which makes a certain association between part of $e(i)$ and $e(j)$ just as the Figure 1. From the definition of the relationship flow, we can derive the following equation:

$$
f\left(s_{i}(t), R_{i j}(t), s_{j}(t)\right)=0 .
$$




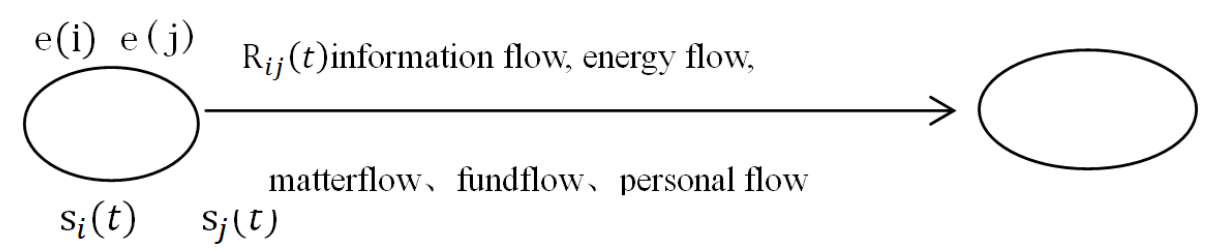

Figure 1. Relationship flow.

Theorem 1: Suppose that under the effect of the system environment, system $Z(n)$ have $m$ levels at the time $t$. Thus, the system input $R f(t)$, the system structure of adjacent level $R_{Z}(t)_{C}$ and $R_{Z}(t)_{C+1}, C=1,2, \ldots, m-1$, and the system behavior $H_{Z}(t)$ has the following relationship: $\psi\left(R f(t), R_{Z}(t)_{c}, R_{Z}(t)_{c+1}\right)=0$.

Theorem 2: Suppose that under the effect of the system environment, relationship flow set $R f_{\text {ord }}(t)$ and the input flow's link which are at the basic level at time $t$ can be denoted as:

$$
\psi\left(R f(t), R f_{\text {Ord }}(t), H_{\text {Or }}(t)\right)=0 .
$$

The Theorems 1 and 2 of the total relationship flow management theory illustrate that under the environment $E(s)$, the organization's operating input flow $R f(t)$, relationship flow set $R f_{\text {ord }}(t)$ at the basic level and organizational behavior $H_{O r}(t)$ affect each other according to certain rules. For complex systems, its behavior only depend on the input relationship flow and relationship flow set $R f_{\text {ord }}(t)$. From these two theorems, we can clearly know that in a given environment the structure of the system determines the behavior of the system. And if you want to get content system behavior or function which the system designer expects, it must carefully design system's hierarchy and optimize the relationship flow.

\subsection{The Total Relationship Flow Management Model}

By the analysis of the above theorems, based on revealing inherent system-designing principles and logic, Professor F. Lin write the literature [6] describes how to build the total relationship flow management model, denoted as (TRFM): Firstly, define organizational behavior at time $t H_{O r}(t)$, and then determine relationship flow set $R f_{\text {ord }}(t)$ including the designing and implementation of relationship flow; finally delimit and find the relationship flow set $R f(t)$ under under the given $H_{O r}(t)$ and $R f_{\text {Ord }}(t)$.

\section{The Design of Recruitment System}

What is the relationship flow? According the total relationship flow management theory, the substance of relationship flow within the organization is cooperation and communication between the different positions. In HR recruitment system, we can understand the relationship flow as each recruitment activities' corresponding. It means that the recruitment system must be constructed to match the organization's strategic objectives in order to provide strong fresh blood for realizing organization's mission and vision. It also means the corresponding between all aspects of the recruitment process, so that the talents can match vacancies to meet human resource requirements and future needs of talent reserving. Based on the total relationship flow management model, how to build a human resource recruitment system should focus on the following aspects.

Firstly, define the expected behavior of recruitment system $H_{O r}(t)$. Recruitment starts from two basis fundamental work: human resource planning and job analysis. It is conducted by its purpose. Only to determine the number of vacancies and the quality which we need to recruit firstly will HR do better in the following process. The human resource planning is to ensure that organization gets the right people at the right time in the different positions (including personnel quality, quantity, structure and level). And job analysis refers to collect some post's comprehensive information. Job specification is the achievement of this work. Trying our best to do these two tasks is the foundation of recruitment, because they determine the expected behavior of recruitment.

Many companies employing department prefer candidates who have highly educated, elite background and famous enterprises experience to deliberately raise the threshold to beautify their image for employment sector or the company. The reason of these blindly selection is lack of job analysis and right employing concept. These conditions not only delay consequent of the recruitment and increase the turnover rate of talent, but also result in a waste of human resource. Based on the idea of the total relationship flow management theory, we must first 
clear expect behavior, so we should focus on human resource planning and job analysis firstly.

Secondly, determine the appropriate relationship flow set $R f_{\text {Ord }}(t)$ and to ensure them smooth. Scientific enterprise recruitment process involves all the steps which we take in there cruitment activities. These steps are integrated with close contact. Every detail and every step is not to be ignored. If we do, the structure of the recruitment will be affected. The total relationship flow management theory point that the structure is the sum of all kinds of relations. More specifically, system structure is refers to information flow, energy flow, matter flow, fund flow, personal flow and the connection mechanism between the five flow. In the recruitment system, these flow acts as the role of the blood. Human resource planning and job analysis provide information flow and fund flow for channel selection and talent selection; channel selection provide personal flow for talent selection, etc. In order to ensure the system operate effectively, it is important to make sure that the flow is smooth and accuracy.

As shown in Figure 1, the relationship flow from $e(i)$ to $e(j)$ can be information flow, energy flow, matter flow, fund flow, personal flow. But in fact, the information flow account for the most part of the total relationship flow in a recruitment system. However information flow in the process of access and the spread will appear some problem of information distortion and information asymmetry, for instance, the real number of absences and human resource planning statistics don't agree with each other. Thus, how to build perfect information flow is very important. But optimizing the information flow is not the whole. As is known to all, personnel flow is the information flow carrier. If only to optimize information flow and ignore the personnel flow, the system could not achieve the optimal structure. Therefore, in order to ensure information flow smooth, we still need to optimize the personnel flow configuration. In the entire recruitment process, it is not enough to rely on the power of the HR department alone. From the Figure 2, we can know that there are relationships between the two adjacent level. So the participation of manager and supervisor from other department in which it has job vacancy is also necessary. The supervisor from department of job vacancies can accurately determine the specific demand for human resources and can assist HR department to do job analysis and give the appropriate comments and suggestions in the link of talent selection.

Realizing relationship flow requires a certain cost. These costs include the resources consumed, time and opportunities, capital investment, construction and deployment of hardware, personnel training, etc. For recruitment system, how to choose the way of fund flow must be considered. Today, Internet is so advanced that online recruitment by virtue of low cost, wide spread is preferred by HRs. But online recruitment is not a panacea and the recruitment of technicians and management staff is not obvious effective in this way. It shows that we must consider comprehensively.

Thirdly, pay attention to the time delay. The relationship flow $R_{i j}(t)$ from $e(i)$ to $e(j)$ usually requires a certain amount of time. This period of time is called delay time of $R_{i j}(t)$. Because of the existence of the delay time, the change of general relationship flow need some time. The time delay usually can have a significant impact for the organization behavior. To the recruitment system, how to control the time delay in the recruitment process is extremely important which is also a factor. When some enterprises especially large enterprises recruit managers, time interval between the adjacent two interviews is so long or the whole process lasts one month. Under this situation, many candidates can't accept such long time delay. Then they will choose to give up. So the time delay between factor in the recruitment system needs reasonable designed to make it within a certain range.

Fourthly, maintain the recruitment system. After the recruitment system according to the total relationship flow management model is designed, how do we maintain the recruitment system? We know that behavior of complex systems only depend on the input relationship flow and relationship flow set $R f_{\text {ord }}(t)$ (the total relationship flow), especially the relationship cycle in the system. Thus, individual behavior actually affects the total relationship flow. In order to eliminate the uncertainty and randomness of individuals and departments, rules and regulations of the recruitment system must be established.

In addition, the designed recruitment system is not constant. With the change of structural frame and organi-

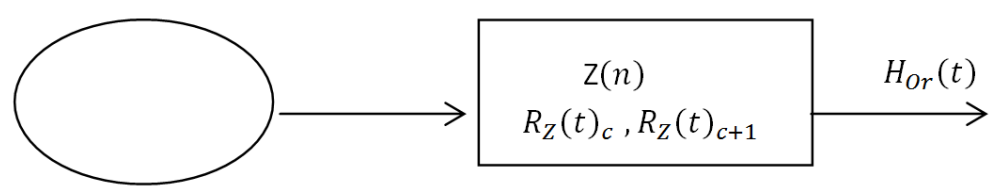

Figure 2. The association between the two adjacent level. 
zation or relocating, the total relationship flow in recruitment system is also to be followed by changes. At this time, we should design the relationship flow again. According to the changes of $H_{O r}(t), R f(t)$ and $R f_{\text {Ord }}(t)$ adjust the frame of relationship flow. Therefore, the human resources department should pay close attention to the external environment and internal organizational changes.

\section{Conclusions}

The recruitment system is composed of a series of workflows which interact and mutual influence with each other. Existing research about recruitment system focuses on how to combine qualitative and quantitative analysis to achieve scientific decisions. However, systems theory states that local optimum does not represent the overall optimum. Just paying attention to the selection of personnel and scientific methods rather than to internal coordinating in the human resources department, will lead the recruitment ineffective.

The total relationship flow management aims at better understanding and controlling the systems' problems. This paper under the guidance of the theory, by determining the recruitment system behavior $H_{O r}(t)$ and the appropriate relationship flow set $R f_{\text {Ord }}(t)$, and focusing on the time delay and system maintenance gives some advice on building the recruitment system, hoping to expand the existing theoretical research in the field and to provide some management implications for managers.

\section{References}

[1] Han, Y.J. (2007) Application of AHP in the Recruitment of Human Resources. Contemporary Finance.

[2] Bertalanffy, L.V. (1950) An Outline of General System Theory. British Journal of Philosophy of Science, 1, 134-164. http://dx.doi.org/10.1093/bjps/I.2.134

[3] Zhang, X.B. (2009) The Review and Research about Human Resource Management System and Organizational Performance. Soft Science, 3, 106-110.

[4] Lin, F.Y. and Wu, J.Z. (1997) General Systems Theory and Its Application Structure. Journal of Systems Engineering, 12, 1-10.

[5] Lin, F.Y. and Cheng, T.C.E. (2007) The Structural Theory of General Systems Applied in Management: The Total Relationship Flow Management Theorems. The International Journal of General Systems, 36, 673-681. http://dx.doi.org/10.1080/03081070701265216

[6] Lin, F.Y., Cheng, T.C.E., Cheng, H., Liang, X.H. and Zhao, J.N. (2013) Developing an Organization Design Framework Based on the Total Relationship Flow Management Theorems. IEEE Transactions on Systems, Man, and Cybernetics: Systems, 43, 1-11. 
Scientific Research Publishing (SCIRP) is one of the largest Open Access journal publishers. It is currently publishing more than 200 open access, online, peer-reviewed journals covering a wide range of academic disciplines. SCIRP serves the worldwide academic communities and contributes to the progress and application of science with its publication.

Other selected journals from SCIRP are listed as below. Submit your manuscript to us via either submit@scirp.org or Online Submission Portal.
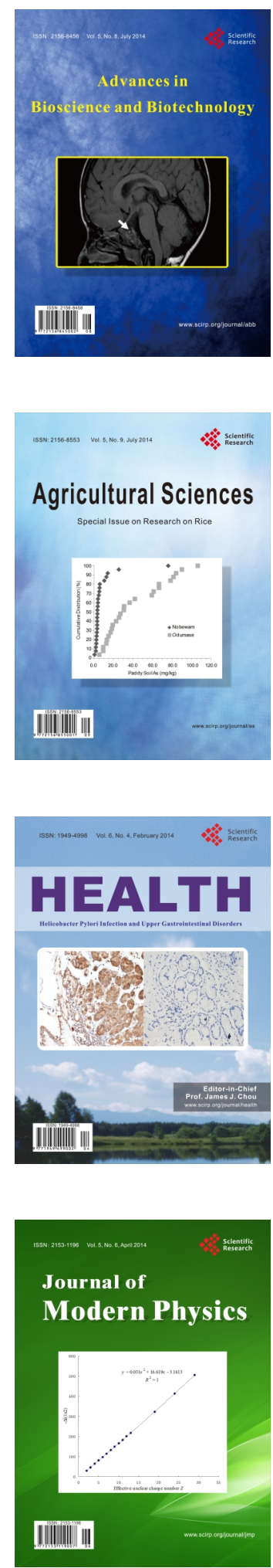
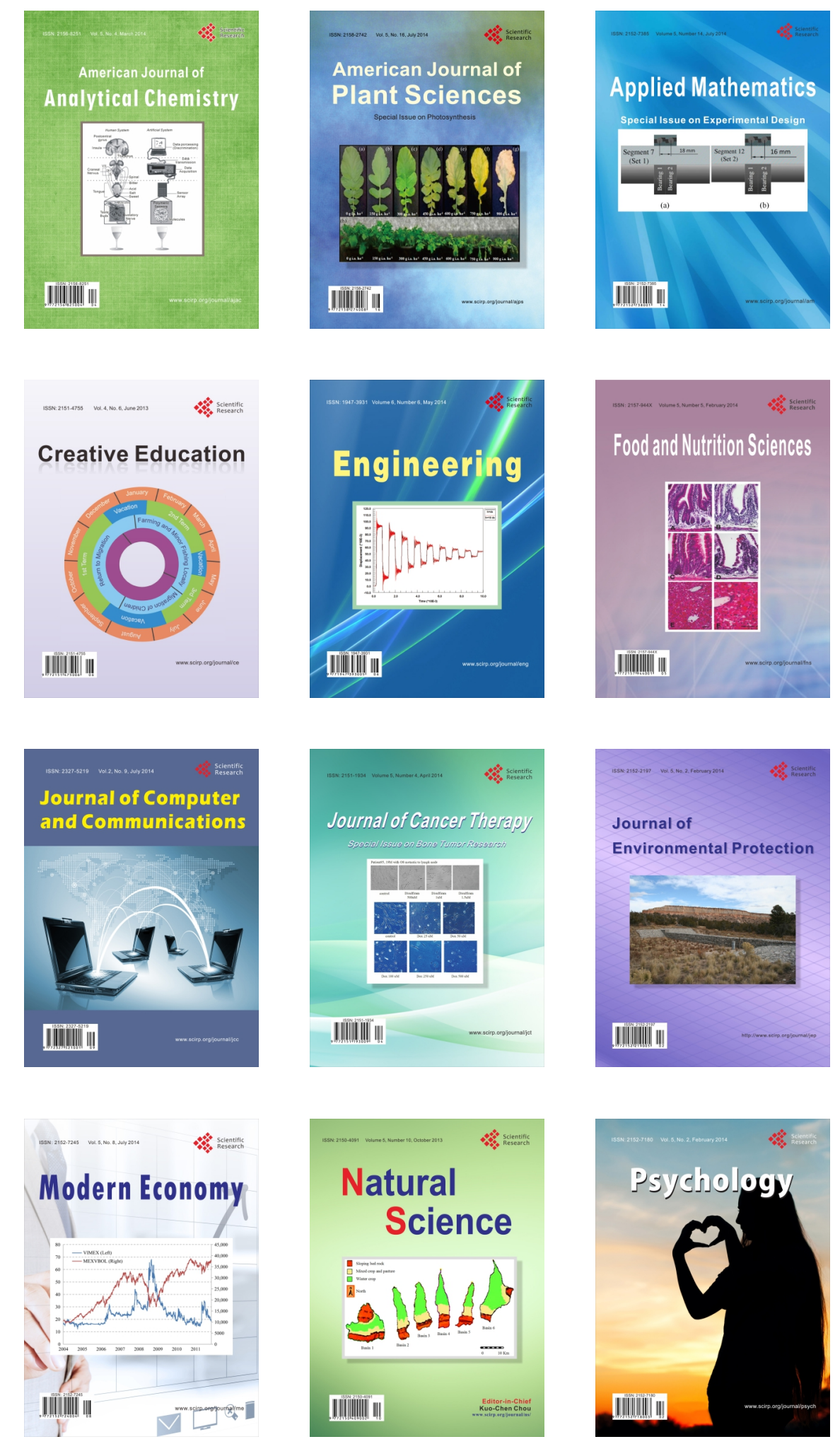